\title{
Experimental methods to determine the hydraulic conductivity
}

\author{
Mihaela-Amalia Diminescuํ, Gabriela-Elena Dumitran ${ }^{1, *}$, and Liana-Ioana Vuţă ${ }^{1}$, \\ ${ }^{1}$ UPB, Hydraulic, Hydraulic Machines and Environmental Engineering Department, 313 Splaiul Independenţei, 060042 \\ Bucureşti, Romania
}

\begin{abstract}
Hydraulic conductivity is one of the most variable and yet an essential parameter in environmental engineering, in estimation of contaminant travel time in soils and groundwater. Also, it is one of the most difficult parameters to determine, and directly affects the quantity of water that will flow. Hydraulic methods for estimation the hydraulic conductivity can be either laboratory methods or in-situ methods. This study presents five laboratory methods which can be used to estimate the hydraulic conductivity of soils: variable-head permeameter - Kamenski; simplified permeameter; constant-head Cromer permeameter, in two working configurations; constant-head permeameter connected to piezometric tubes and a variable-head permeameter. Although these methods require more labour than other available methods, e.g. the correlation methods, they are relatively fast and not expensive. For the porous material used in the experiments, the most accurate values of the hydraulic conductivities were obtained with the constant-head permeameter connected to piezometric tubes.
\end{abstract}

\section{Introduction}

The physical property which denotes the capacity of a material to allow water flow, in the presence of an applied hydraulic gradient, through pore spaces and fractures is the hydraulic conductivity [1].

This property, noted $K$, is influenced by the density and viscosity of the fluid, the saturation degree and the permeability of the material. Hydraulic conductivity is defined by Darcy's law as the ratio of the Darcy's velocity to the applied hydraulic gradient [1].

In order to determine the hydraulic conductivity of soils, $K$, correlation methods or hydraulic methods can be used.

The correlation methods estimate the $K$-value using a preset relationship with a soil property which is easy to determine (e.g. texture) and have the major advantage of being simpler and quicker than direct determination of the hydraulic conductivity [2-3].

The hydraulic methods consist in establishing the desired flow conditions in the soil and using a proper formula, based on the Darcy's law and the boundary conditions.

The $K$-value is calculated based on the values of hydraulic head and discharge observed under the imposed conditions.

Hydraulic methods, even if implies more effort than the correlation methods, have the advantage of excluding the

uncertainties associated with the linking of a soil property to the hydraulic conductivity, and also are simple, inexpensive and relatively fast.

Both methods have comparable weaknesses in terms of variability and representativeness.
Also, there are many relations available for estimating the hydraulic conductivity of soils [4-5], but Kozeny-Carman relation, proposed by Kozeny and improved by Carman is the most used [6]:

$$
K=C_{F} \frac{1}{A^{2}} \frac{\gamma}{\mu \cdot \rho_{m}} \frac{e^{3}}{1+e}
$$

where $K$ is the hydraulic conductivity, $(\mathrm{m} / \mathrm{s}), e$ is void ratio of soils, which is dimensionless; $C F$ is a dimensionless shape constant, with a value about $\mathrm{CF} \approx 0.2 ; A$ is the specific surface area of particles $\left(\mathrm{m}^{2} / \mathrm{g}\right)$; $\gamma=$ unit weight of fluid $\left(\mathrm{N} / \mathrm{m}^{3}\right) ; \rho_{m}\left(\mathrm{~kg} / \mathrm{m}^{3}\right)$ is particle density of soil; $\mu\left(\mathrm{N} \cdot \mathrm{s} / \mathrm{m}^{2}\right)$ is fluid viscosity.

This study aims to compare multiple experimental methods for determining the hydraulic conductivity with the exact value calculated using Carman formula and to establish which of these methods is the most accurate. Thus, five experimental methods are analyzed:

- Variable-head permeameter - Kamenski;

- Simplified permeameter;

- Constant-head Cromer permeameter, in two working configurations;

- Constant-head permeameter connected to piezometric tubes;

- Variable-head permeameter.

\section{Methodology}

The soil used for the experiments belongs to coarse-grain soils, medium and fine sand.

By using the Carman formula (eq. 1), the hydraulic conductivity of the analysed soil was found to be 0.26

\footnotetext{
"Corresponding author: gabriela.dumitran@upb.ro, mihaela.diminescu@yahoo.com
} 
$\mathrm{cm} / \mathrm{min}$.

The following characteristics of the soil have been determined into laboratory: the density of the solid matrix of $2580 \mathrm{~kg} / \mathrm{m}^{3}$, bulk density of $1650 \mathrm{~kg} / \mathrm{m}^{3}$, total porosity $37 \%$, effective porosity $30 \%$ and void ratio 0.563 .

These material's physical properties are necessary and represents the first step of the experiment.

The density of the solid matrix $\rho_{\mathrm{s}}$ is given by the ratio of dry sand mass, $m_{s}$ and the volume of solid particles $V_{s}$ (for five sand samples):

$$
\rho_{s}=\frac{m_{s}}{V_{s}}\left[\frac{k g}{m^{3}}\right]
$$

Bulk density represents the ratio between dry sand mass, $m_{s}$ and the total volume of sand sample $V_{t}$ :

$$
\rho_{d}=\frac{m_{s}}{V_{t}}\left[\frac{\mathrm{kg}}{\mathrm{m}^{3}}\right]
$$

Total porosity, $n$, is defined as ratio of the volume of void space and the total volume of the sample and it was calculated as the average of the five samples analysed:

$$
n=\frac{V_{p}}{V_{t}}[\%]
$$

Effective porosity, $n_{e}$, is quantified by the ratio between the volume of free water released by a saturated porous medium under the effect of complete drainage (free drainage, gravity drainage) and its total volume.

$$
n_{e}=\frac{V_{e f f}}{V_{t}}[\%]
$$

Void ratio, characterizes the compactness of soils, is noted $e$, and is given by the ratio of the volume of voidspace, $V_{p}$, and the volume of solids, $V_{s}$.

$$
e=\frac{V_{p}}{V_{S}}
$$

Void ratio and porosity are inter-related to each other as follows [3]:

$$
n=\frac{e}{e+1}
$$

\subsection{Variable head permeameter - Kamenski}

Variable-head permeameter Kamenski (Figure 1) is used for hydraulic conductivities below $10^{-5} \mathrm{~m} / \mathrm{s}$. The flow is estimated using the volumetric method.

In order to determine the hydraulic conductivity, the level difference between the free water surface in the Kamenski tube, $H_{0}$ and the free water surface of the tank $H(t)$, respectively $h i(t)$ must be measured, as well as the time, $t_{i}$.

For optimal conditions during the experiment, the velocity through soil sample is limited (by the validity of Darcy's law which can be applied only for laminar groundwater flow), thus the level in the reservoir must be kept as high as possible.
The height of the soil sample column in the experimental permeameter is $L=17 \mathrm{~cm}$. Hydraulic conductivity is given by:

$$
K=2,3 \frac{L}{t_{i}} \lg \frac{H_{0}}{H_{0}-h_{i}}
$$

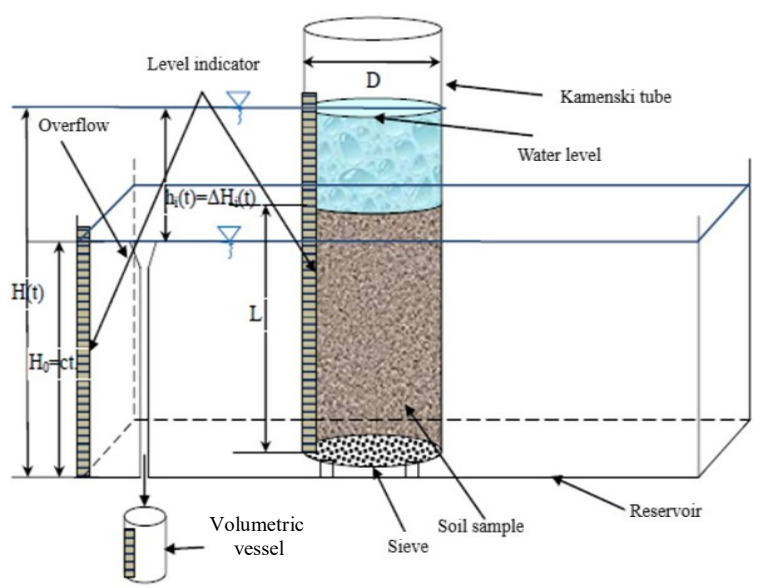

Fig. 1. Kamenski permeameter.

The procedure is as follows: a soil sample of $L=17$ $\mathrm{cm}$ height is placed into the permeameter and the tube is introduced in the tank so that the soil saturates from the bottom up. The permeameter is fed with water. The experiments were performed for three samples of sand, having the same characteristics, and the hydraulic conductivity was calculated by measuring the level differences between the free surface of the Kamenski tube, $H_{0}$ and the free water surface of the tank $H(t)$, respectively $h_{i}(t)$.

\subsection{Simplified permeameter}

The simplified permeameter contains a transparent plastic tube (Figure 2) with one end covered by a filter. The cylinder is placed in a water tank.

The sand tested should be $100 \%$ saturated with water and left to compact naturally to simulate an underground environment and. Thus, during the experiment, a compaction and saturation protocol involving several steps must be followed (Figure 3).

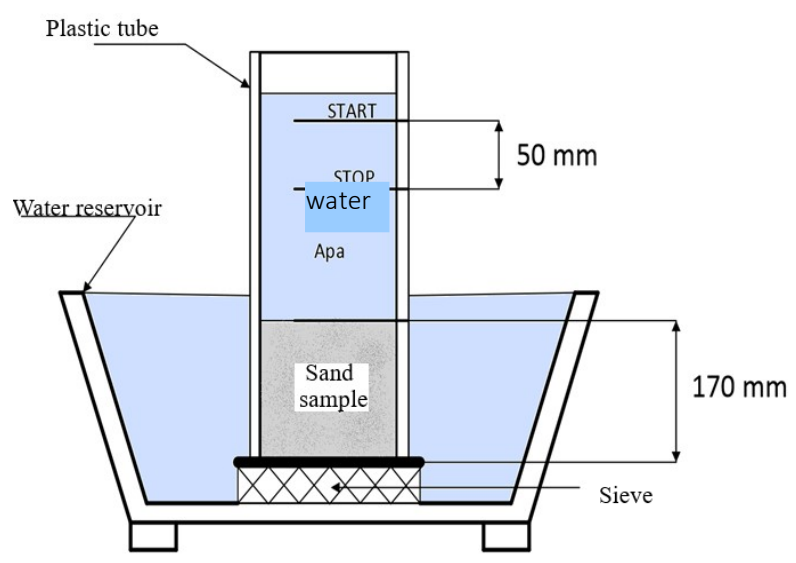


Fig. 2. Simplified permeameter.

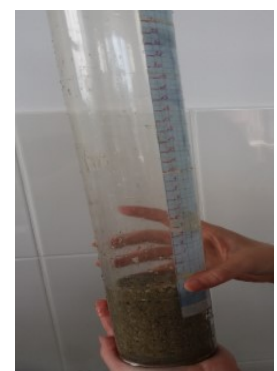

a) step 1

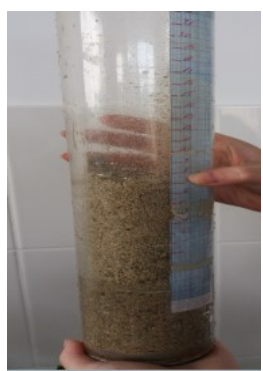

b) $\quad$ step 2

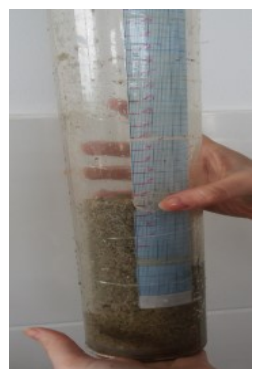

c) $\quad$ step 3 $\mathrm{l}=170 \mathrm{~mm}$
Fig. 3. The procedure for sand compaction and saturation.

Then, a column of $17 \mathrm{~cm}$ of sand is added into the cylinder and compacted. The cylinder is then inserted into the container and the sand is filled with water from the bottom up, to remove the air. Next, water is carefully poured into the top of the cylinder, above the top reference mark. Water level decreases and the time needed to travel $50 \mathrm{~mm}$, the distance highlighted on the cylinder by Star and Stop notations, is measured. The water temperature during the testing was $20^{\circ} \mathrm{C}$.

In this case, the hydraulic conductivity is obtained by using the permeability diagram presented in Figure 4.

Depending on the time of the infiltration of water and on the temperature of water, the hydraulic conductivity value, in $\mathrm{m} / \mathrm{s}$, is read from the diagram.

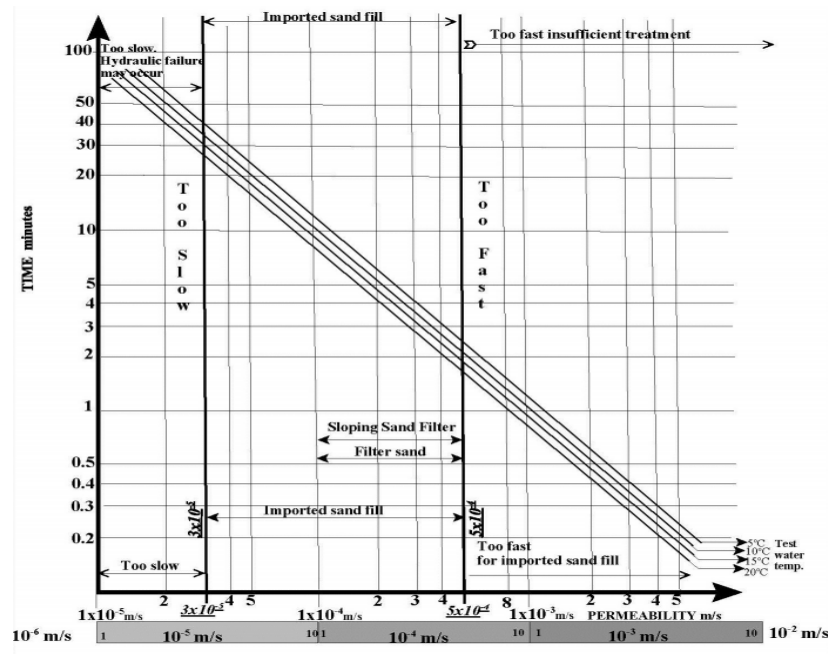

Fig. 4. Permeability diagram [7].

\subsection{Constant-head Cromer permeameter, in two working configurations}

The Cromer permeameter contains a graduated water tank (Figure 5) and a feeding tube, which together assures a constant water depth in the test orifice. For permeability testing, the tank is provided with a distribution tube and the assembly is lowered vertically into a test orifice (containing previously added water) until the bottom open end of the tube is just immersed.
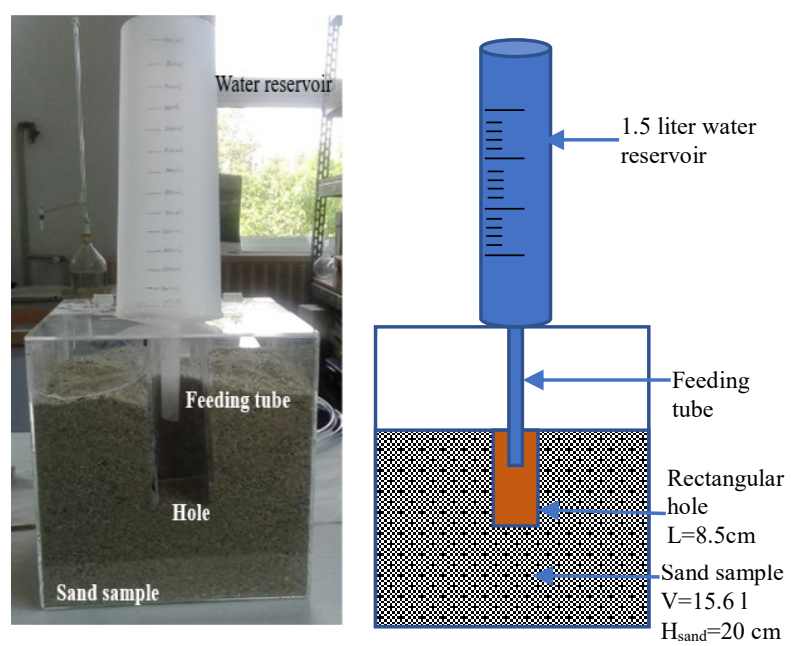

Fig. 5. Cromer permeameter - first working configuration, photo and schematic view.

Two working configurations of this permeameter were used to determine the hydraulic conductivity of the test samples (Figures 5 and 6). The differences between the two working configuration are: the length of the feeding tube, in the first case $l=8.5 \mathrm{~cm}$ while in the second case $l=16 \mathrm{~cm}$ and the shape of the hole in the sand sample, in the first case - rectangular and in the second one cylindrical.
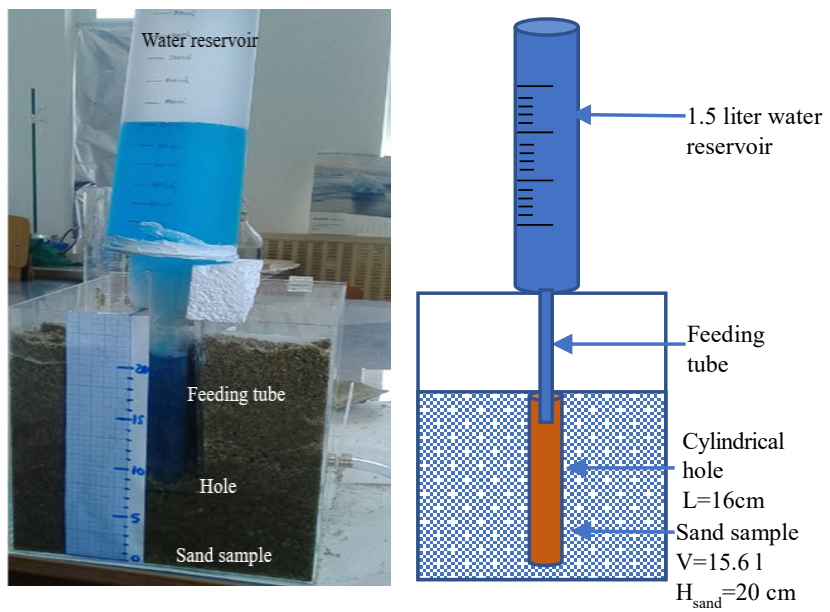

Fig. 6. Cromer permeameter - second working configuration, photo and schematic view.

Hydraulic conductivity is found by measuring the fluid flow through cross-sectional area over time. It can be used for hydraulic conductivities ranging from 0.0005 to $10 \mathrm{~m} /$ day.

Depending on the distance between the bottom of the test orifice and the "impermeable" layer, the permeability calculation is based on one of the following equations:

$$
\begin{gathered}
K=\frac{4,4 \cdot Q \cdot\left\{0,5 \cdot \sinh ^{-1}\left(\frac{H}{2 r}\right)-\left[\left(\frac{r}{H}\right)^{2}+0,25\right]^{0,5}+\frac{r}{H}\right\}}{2 \pi H^{2}} \\
K=\frac{3 \cdot Q \cdot \ln \left(\frac{r H}{r}\right)}{\pi H(3 H+2 S)}
\end{gathered}
$$


where: $Q$ - water flow at a constant infiltration rate, $H$ depth of water in the test orifice, $r$ - test orifice radius, $S$ - depth of the impermeable layer.

Equation 9 is valid for the cases when the distance from the bottom of the test orifice to the impermeable layer is twice the depth of the water in the orifice $(S>$ $2 H$ ), otherwise the equation 10 is used.

For the first working configuration, a feeding tube of length $l=8.5 \mathrm{~cm}$ was used and two measuring designs were used:

- First case:

The water column in the test hole has a constant height, $H=7 \mathrm{~cm}$; the distance between the bottom of the test orifice and the impermeable surface is $S=10 \mathrm{~cm}$; the radius of the inscribed circle in the rectangular section of the hole $r=3 \mathrm{~cm}$.

- Second case:

The water column in the test hole has a constant height, $H=14 \mathrm{~cm}$; the distance from the bottom of the test orifice to the impermeable surface, $S=8 \mathrm{~cm}$ and the radius of the inscribed circle in the rectangular section of the orifice $r=1.35 \mathrm{~cm}$.

For the second working configuration, a feeding tube of $16 \mathrm{~cm}$ length (1) is used, and the rest of the characteristic dimensions are: constant height of the water column $(H)$ in the test hole of $8 \mathrm{~cm}$; the distance from the bottom of the test port to the impermeable surface $S=7 \mathrm{~cm}$ and the radius of the inscribed circle in the rectangular section of the orifice is $r=1.35 \mathrm{~cm}$.

\subsection{Constant-head permeameter, connected to piezometric tubes}

This type of permeameter (Figure 7) is mainly used to determine the permeability of pebbles and sand. The permeability coefficient is calculated based on Darcy's law, using the equation:

$$
K=\frac{v \cdot H}{t \cdot A \cdot \Delta h}
$$

where: $v$ - the volume of water collected $(\mathrm{ml}) ; H$ - the height of the material column between the two piezometric tubes $(\mathrm{cm}) ; t$-time needed to collect the water (s); $A$ - the cross-section area of the permeameter $\left(\mathrm{cm}_{2}\right)$ and $\Delta \mathrm{h}$ - the pressure difference, $\Delta h=h_{1}-h_{2}$.

Firstly, the mass of soil in the permeameter was determined, $m_{s}=740 \mathrm{~g}$, and a filter disc was placed at the bottom of the permeameter. The volume of the sample was calculated, based on the height of the solid sample $(l=27 \mathrm{~cm}), V=530.14 \mathrm{~cm}^{3}$. The flow was computed using the volumetric method and the pressure difference was read using the piezometric tubes.
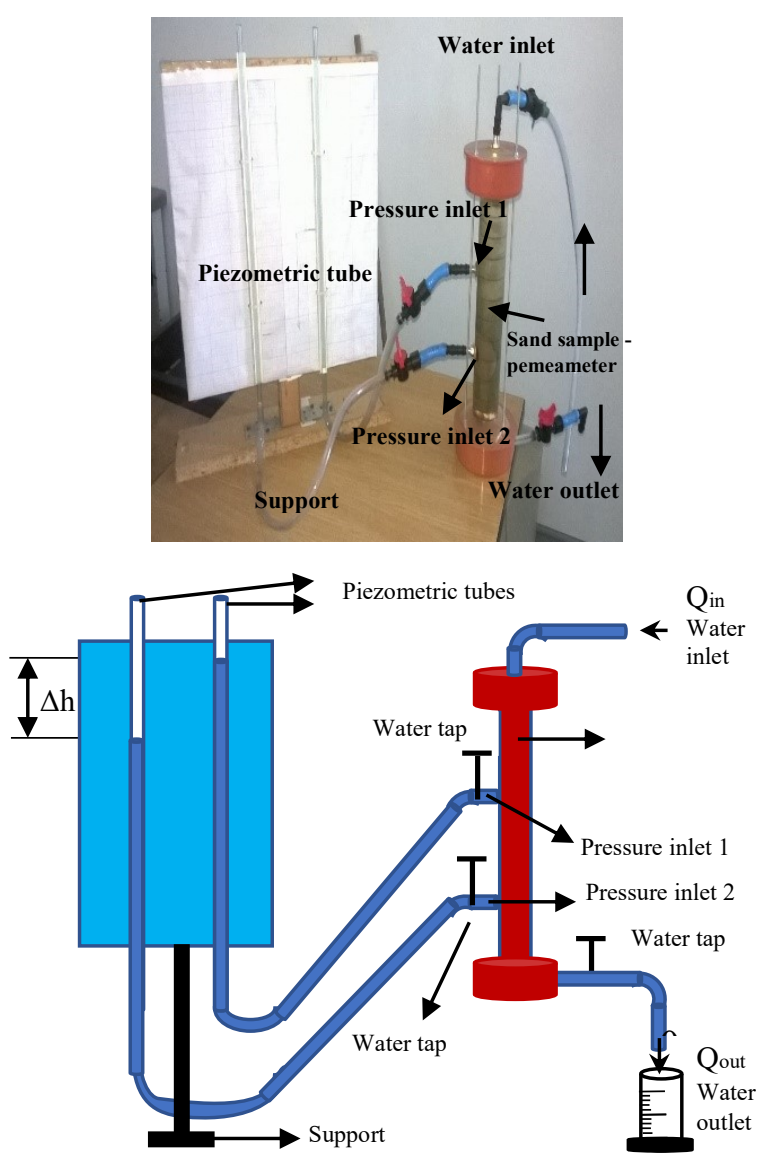

Fig. 7. Constant-head permeameter with piezometric tubes, photo and schematic view.

\subsection{Variable-head permeameter}

This type of permeameter is used to determine the permeability coefficient for fine granular materials (Figure 8). In this case, also, in order to calculate the permeability coefficient, Darcy's law is used, but the calculus is not as direct as in the case of constant-head permeameter because the hydraulic gradient varies over time.

The relation used to determine the permeability coefficient for the variable-level permeameter is:

$$
K=\frac{a \cdot l}{A \cdot t_{1}} \cdot \ln \frac{h_{0}}{h_{1}}
$$

where: $a$ - cross-sectional area of the feeding orifice, $\left(0.785 \mathrm{~cm}^{2}\right), l$ - the length of the column of porous material, $(27 \mathrm{~cm}), A=$ cross-sectional area of the column of porous material studied, $\left(19.63 \mathrm{~cm}^{2}\right)$ and $t_{l}$ - the time required to decrease the level from $h_{0}$ to $h_{1}(\mathrm{~s})$, with $h_{0}-$ the loss of level over the length of the material column at $t=0$ and $h_{l}=$ the loss of level over the length of the material column at $t=t_{l}$. In this case, the soil mass is $m_{s}$ $=740 \mathrm{~g}$ and the volume of the soil column in the permeameter is $V=530.14 \mathrm{~cm}^{3}$. 

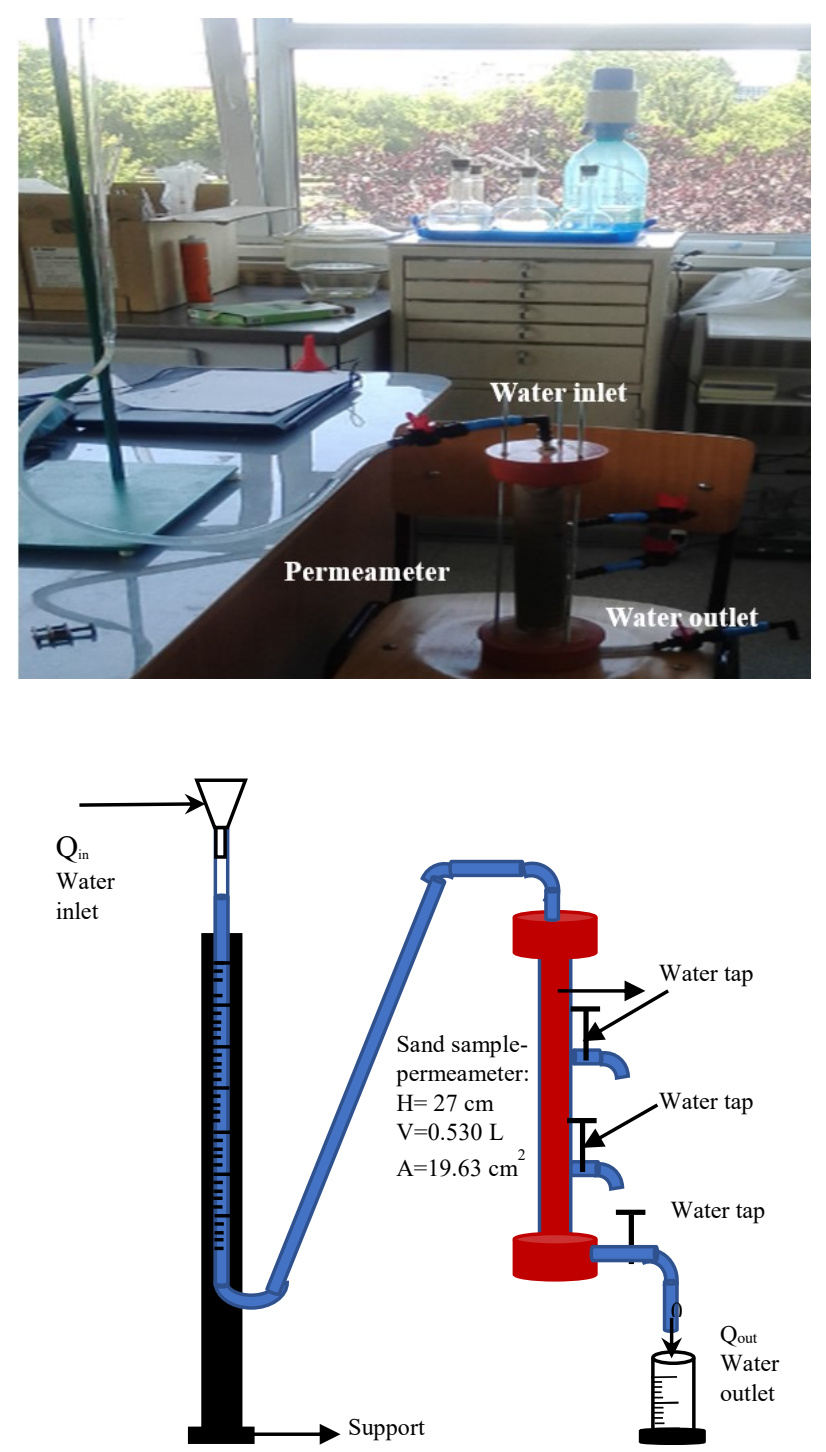

Fig. 8. Variable-head permeameter, photo and schematic view.

The permeability is determined by measuring the volume of fluid flowing through cross-sectional area over a given time and determining the water flow rate into the interstice of the graduated tank.

\section{Results and discussion}

The experimental results obtained with the five different types of permeameter are presented further on.

\subsection{Variable-head permeameter - Kamenski}

Three experiments using the variable-head permeameter - Kamenski have been realized, using the same porous material. The values obtained for the hydraulic conductivity varied for the first experiment from 0 to $0.24 \mathrm{~cm} / \mathrm{min}$, for the second experiment from 0 to 0.26 $\mathrm{cm} / \mathrm{min}$ and for the last experiment from 0 to 0.25 $\mathrm{cm} / \mathrm{min}$. The experimental results and the medium values of the hydraulic conductivity are presented in Figure 9.

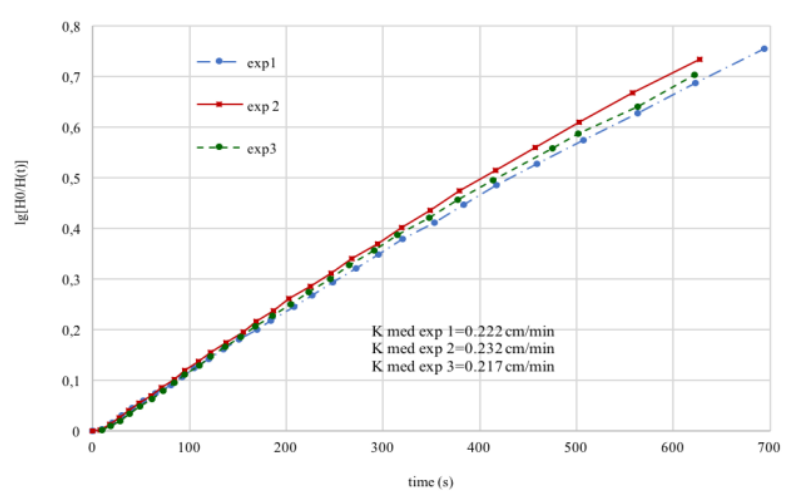

Fig. 9. Correlation curves - Kamenski permeameter.

\subsection{Simplified permeameter}

For the simplified permeameter, the values of hydraulic conductivity obtained varies from 0.21 to $0.25 \mathrm{~cm} / \mathrm{min}$, and the medium value is $0.23 \mathrm{~cm} / \mathrm{min}$. Figure 10 shows the results in this case.

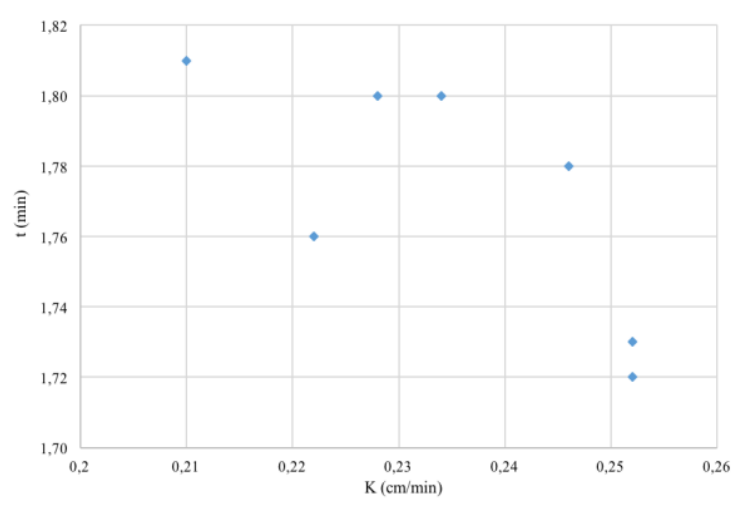

Fig. 10. Values of hydraulic conductivity found with the simplified permeameter.

\subsection{Constant-head Cromer permeameter, in two working configurations}

For first configuration of constant-head Cromer permeameter, two different designs were used, and for each of them two experiments were undergone. Thus, for the first design the values of hydraulic conductivity varied during the first experiment from 0.078 to 0.305 $\mathrm{cm} / \mathrm{min}$, with a mean value of $0.263 \mathrm{~cm} / \mathrm{min}$ and during the second experiment from 0.217 to $0.37 \mathrm{~cm} / \mathrm{min}$, with a medium value of $0.272 \mathrm{~cm} / \mathrm{min}$. When using the second design, the values of hydraulic conductivity varied during the first experiment from 0.021 to 0.355 $\mathrm{cm} / \mathrm{min}$, with a mean value of $0.236 \mathrm{~cm} / \mathrm{min}$, while during the second experiment the values ranged from 0.212 to $0.264 \mathrm{~cm} / \mathrm{min}$, with a medium value of 0.242 $\mathrm{cm} / \mathrm{min}$.

The second working configuration lead to following values of the hydraulic conductivity: 0.071 to 0.324 $\mathrm{cm} / \mathrm{min}$ with a medium of $0.255 \mathrm{~cm} / \mathrm{min}$ for the first experiment and from 0.0503 to $0.29 \mathrm{~cm} / \mathrm{min}$ with a medium of $0.208 \mathrm{~cm} / \mathrm{min}$ for the second one. 
The results are presented in Figure 11.

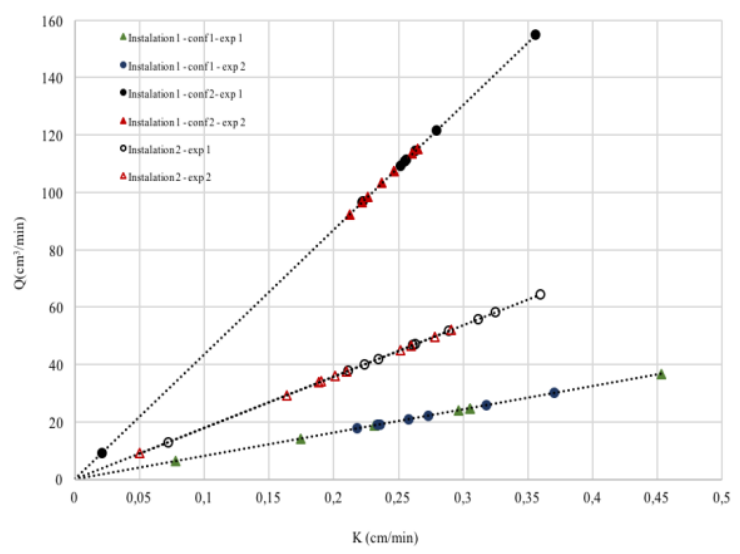

Fig. 11. Values of hydraulic conductivity found with Cromer permeameter.

\subsection{Constant-head permeameter, connected to piezometric tubes}

Three experiments have been undergone with this permeameter, and the values of hydraulic conductivity varied as follows:

- for the first experiment from 0.231 to $0.306 \mathrm{~cm} / \mathrm{min}$ and the mean value is $0.259 \mathrm{~cm} / \mathrm{min}$;

- for the second experiment from 0.244 to $0.377 \mathrm{~cm} / \mathrm{min}$, with a medium value of $0.292 \mathrm{~cm} / \mathrm{min}$;

- for the third one from 0.238 to $0.312 \mathrm{~cm} / \mathrm{min}$ and a medium value of 0.295 .

The results are shown in Figure 12.

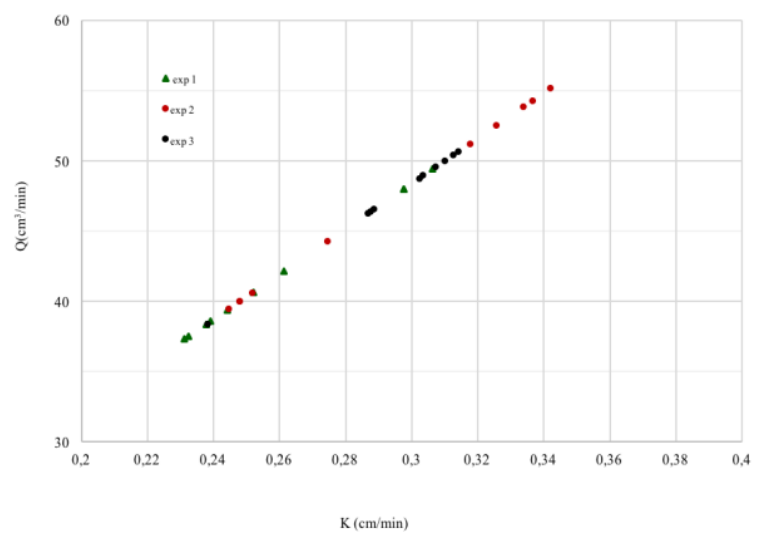

Fig. 12. Values of hydraulic conductivity found using the constant-head permeameter.

\subsection{Variable-head permeameter}

Using the variable-head permeameter, the values of the hydraulic conductivity varied from 0 to $0.719 \mathrm{~cm} / \mathrm{min}$, with an average value of $0.288 \mathrm{~cm} / \mathrm{min}$. The results are presented in Figure 13.

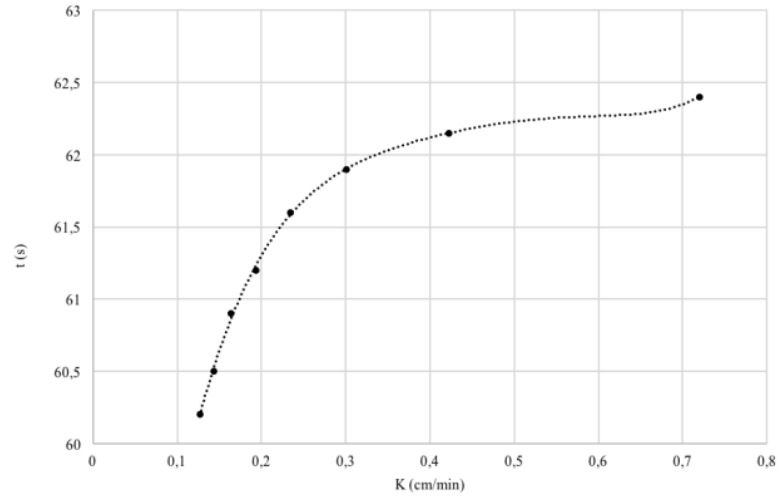

Fig. 13. Values of hydraulic conductivity found using the variable-head permeameter.

\subsection{Short statistical analysis of experimental results}

In order to analyse the results, the experimental methods were numbered from 1 to $14: 1$ to 3 the three experiments using the variable-head permeameter Kamenski; 4 - simplified permeameter; 5 to 10 the six experiments using constant-head Cromer permeameter; 11 to 13 the three experiments using a constant-head permeameter connected to piezometric tubes and 14, the variable-head permeameter.

Figure 14 summarizes the results.

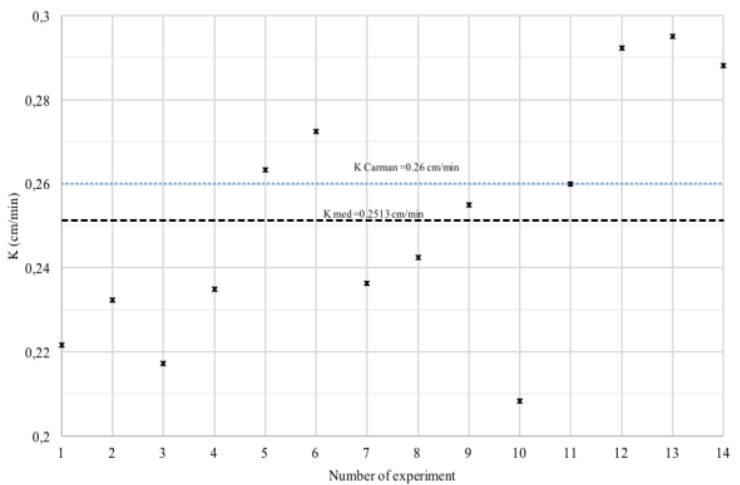

Fig. 14. Values of hydraulic conductivity found during the 14 experiments.

The results obtained were evaluated by computing the mean square deviation. In probability and statistics, the standard deviation is a measure of the dispersion of a set of values and is usually noted with the Greek letter $\sigma$. The standard deviation is defined as the root-meansquare (RMS) deviation of the values from their mean, or as the square root of the variance. For the experimental values obtained the standard deviation is 0.0273 and the Gaussian curve is presented in figure 15 [8]. It describes the probability density over the range of the distribution and the abscissa and the ordinate values are given by:

$$
X=\frac{x-\mu}{\sigma}
$$




$$
f(x)=\frac{1}{\sigma \sqrt{2 \pi}} e^{-\frac{(x-\mu)^{2}}{2 \sigma^{2}}}
$$

where: $x$ are the experimental values of the hydraulic conductivity and $\mu$ is the average hydraulic conductivity.

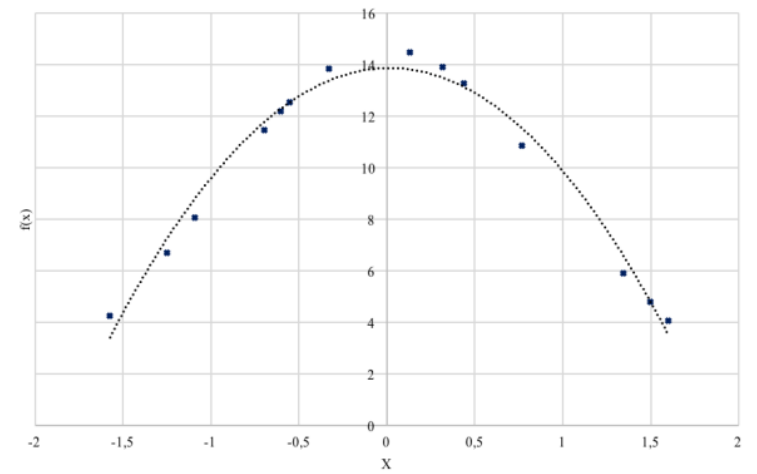

Fig. 15. Gaussian curve of the experimental values found.

Comparing the mean value of the hydraulic conductivity found based on experimental data, of $0.2513 \mathrm{~cm} / \mathrm{min}$, with the value given by Carman's relation, of $0.26 \mathrm{~cm} / \mathrm{min}$, it can be observed a deviation of approximately $8 * 10^{-3}$, with an error of 3,3\%.

The closest value of hydraulic conductivity was found by using the constant-head permeameter connected to piezometric tubes of $0.2599 \mathrm{~cm} / \mathrm{min}$, leading to a percentage error of 0.01 . The most remote value was found during the second experiments with the Cromer permeameter - second working configuration: $0.208 \mathrm{~cm} / \mathrm{min}$, which leads to an error of approximately $20 \%$. The error corresponding to the first experiments with the Cromer permeameter - second working configuration is $1.93 \%$. For the rest of experiments done with the Cromer permeameter - first working configuration, the values of percentage error are: 1.28, $4.75,9.15,6.8$, and $1.9 \%$, while the errors corresponding to the values of hydraulic conductivity found with simplified permeameter and variable-head permeameter are of approximately $10 \%$.

The worst overall values were the ones found when using the Kamenski permeameter, the experimental values found being: $0.221,0.232$ and $0.217 \mathrm{~cm} / \mathrm{min}$, with corresponding percentage errors of 14.8, 10.6 and $16.4 \%$.

\section{Conclusions}

This study aims to compare multiple experimental methods for determining the hydraulic conductivity: a variable-head permeameter - Kamenski, a simplified permeameter, a constant-head Cromer permeameter, in two working configurations, a constant-head permeameter connected to piezometric tubes and a variable-head permeameter.

The experimental devices used are handmade, inexpensive, portable, easy to use and readily changeable for other types of studies. This is the reason way the present study used just one type of porous material, a non-cohesive one, even if, according to the standards [9], the constant-head permeameter is used for non-cohesive materials while the variable-head permeameter for cohesive materials.

Comparing each of the experimental values of hydraulic conductivity found with the value given by Carman's equation, it can be noticed that the most accurate result was found by using the constant-head permeameter connected to piezometric tubes, while the most imprecise is the one given by Kamenski permeameter.

This work has been funded by University Politehnica of Bucharest, through the "Excellence Research Grants" Program, UPB - GEX 2017. Identifier: UPB- GEX2017, Ctr. No. 8 /25.09.2017 ( SI - Acvi)"

\section{References}

1. R.J. Oosterbaan, H.J. Nijland, H.P. Ritzema, Drainage Principles and Applications, I.L.R.I., 16, 435 (1994)

http://citeseerx.ist.psu.edu/viewdoc/download?doi=1 0.1.1.694.8962\&rep=rep1\&type $=$ pdf

2. H.T. Hwang, S.W. Jeen, A.A. Suleiman, K.K. Lee, Water, 9, 942 (2017) https://doi.org/10.3390/w9120942

3. D. K. Todd, L.W. Mays, Groundwater hydrology (John Wiley \& Sons Inc., 2005)

4. R.P. Chapuis, D.E. Gill, K. Baass. Can. Geotech. J., 26, 614 (1989) https://doi.org/10.1139/t89-074

5. R. Xingwei, Z. Yang, D. Qinglu, K. Jianyu, L. Dexian, W. Debin, Eng. Geol., 213, 89 (2016) https://doi.org/10.1016/j.enggeo.2016.08.017

6. P.C. Carman, Trans. Inst. Chem. Eng., 15, 150 (1937)

https://doi.org/10.1016/S0263-8762(97)80003-2

7. http://www.williamccromer.com

8. M. I. Ribeiro, I.S.R., Instituto Superior Tcnico (2004)

9. STAS $1913 / 6-76$ 